\title{
Temporal Multi-level Coordination Techniques Oriented to Regional Water Networks: Application to the Catalunya Case Study
}

\author{
Cong Cong Sun ${ }^{\mathrm{a}}$, Vicenç Puigg,*, Gabriela Cembrano ${ }^{\mathrm{a}, \mathrm{b}}$ \\ ${ }^{a}$ Advanced Control Systems Group at the Institut de Robòtica i Informàtica Industrial \\ (CSIC-UPC), Llorens i Artigas, 4-6, 08028 Barcelona, (Spain). Tel: +34 688041855 \\ ${ }^{b}$ CETaqua, Water Technology Centre at Esplugues 75, 08940, Cornellà de Llobregat, Barcelona
}

\begin{abstract}
In this paper, a multi-layer model predictive control (MPC) with temporal multilevel coordination for regional water supply systems is proposed. First, a multilayer control structure resulting from a functional decomposition of water network is briefly presented. Inside each layer, an MPC based controller is used. Between related layers, a temporal multi-level coordination mechanism is used to generate control strategies which consider objectives and time scales of both layers. The upper layer which is named supply layer works in a daily scale in order to achieve the global management policies for the different reservoirs. The lower layer which is named transportation layer works in a hourly scale and is in charge of manipulating the actuators (pumps and valves) set-point to satisfy the local objectives. The results of the modelling will be applied to the Catalunya Regional Water Network and this paper presents the simulation results based on an aggregate model of this network.
\end{abstract}

Keywords: multi-layer MPC, temporal multi-level strategy, regional water

\footnotetext{
${ }^{*}$ Corresponding author

Email address: vicenc.puig@upc. edu (Vicenç Puig)
} 
network

\section{Introduction}

Complex regional water supply system management is an important research topic because of the significance of water for human beings.

From a functional perspective, a regional water network can be structurally organized into three layers (Ocampo-Martinez et al., 2013):

- Supply layer, composed of water sources, large reservoirs and also natural aquifers.

- Transportation layer, linking water treatment and desalinization plants with reservoirs distributed all over the city.

- Distribution layer, used for meeting consumer demands.

Each of the layers of a regional water network must be operated at different time scale because of the different dynamics they present according to their specified objectives. In general, these layers are often separately operated. What is more, the ecological effect and also sustainable usages of water which are important has seldom been included (Brdys \& Ulanicki, 1994; Cembrano et al., 2005; Ocampo-Martinez et al., 2013). The coordinated operation of different layers in a regional network is one of the main motivations for the research reported in this paper.

In most water systems, the actuators, named valves, turbines, pumps, gates and retention devices, are controlled locally (using simple control laws such as PIDs), i.e., they are controlled by a remote station according to the measurements of sensors connected only to that station. However, a global real-time control (RTC) 
system requires the use of an operational model of the system dynamics in order to compute, ahead in time, optimal control strategies for the actuators based on the current state of the system provided by supervisory control and data acquisition (SCADA) sensors, the current disturbance measurements and appropriate disturbance predictions. The computation of an optimal global control law should take into account all the physical and operational constraints of the dynamical system, producing set-points which cause certain control objectives to be achieved.

MPC has been proven to be one of the most effective and accepted control strategies for the global optimal operational control of large-scale water networks (Ocampo-Martinez et al., 2013). Applications to different large-scale infrastructures as drinking water networks (Brdys \& Ulanicki, 1994), sewer networks (Marinaki \& Papageorgiou, 2005), open-flow channel networks (Overloop, 2006) or electrical networks (Negenborn, 2008) prove the advantages of this technique. One of the main reasons for its success is that once the plant dynamical model has been obtained, the MPC design consists in expressing the desired performance specifications through different control objectives (e.g., weights on tracking errors and actuator efforts as in classical linear quadratic regulation), and constraints on system variables (e.g., minima/maxima of selected process variables and/or their rates of change) which are necessary to ensure process safety and asset health. The rest of the MPC design is straightforward: the given model, constraints and weights define an optimal control problem over a finite time horizon in the future (for this reason the approach is called predictive). This is translated into an equivalent optimization problem and solved on line to obtain an optimal sequence of future control actions. Only the first of these actions is applied to the process, as at the next time step a new optimal control problem is solved, to exploit the infor- 
mation coming from fresh new measurements. In this way, an open-loop design methodology (i.e., optimal control) is transformed into a feedback one.

In recent literature, there is a renewed interest in multi-layer MPC either from industrial practice or from academia (R.Scattolini, 2009; Tatjewski, 2008). This is specially the case when a system is composed of subsystems with multiple time scales as in the case of the regional water networks. A straightforward task of designing and implementing a single centralized control unit is too difficult as discussed in Brdys et al. (2008), because the required long prediction horizon and short control time steps might lead to an optimization problem of very high dimension and under large uncertainty radius. A way to cope with this problem is to apply a hierarchical control structure based on decomposing the original control task into a sequence of different, simpler and hierarchically structured subtasks, handled by dedicated control layers operating at different time scales (Brdys \& Tatjewski, 2005).

The main contribution of this paper is proposing a temporal multi-layer hierarchical MPC scheme for regional water networks, which according to the literature review has never been applied before to this type of water networks. The proposed strategy will coordinate the MPC controllers for the supply and transportation layers by means of a temporal hierarchical sequence of optimizations and constraints going from the upper to the lower layer. The Catalunya Regional Water Network is used as a case study to validate the proposed control scheme.

The paper is organized as follows: Section 2 introduces the control oriented modelling methodology proposed for regional water networks. Then in Section 3, MPC techniques and MPC models for the supply and transportation layers are provided. In Section 4, Multi-layer MPC and temporal multi-level coordination 
techniques are outlined. In Section 5, the formulations of temporal multi-level coordination for regional water network and predicting water demand for regional water network is presented. In Section 6, the considered case study based on the Catalunya Regional Water Network is described. In Section 7, the simulation results of the proposed approach applied to Catalunya Regional Water Network are outlined. Finally, in Section 8, the main conclusions are presented.

\section{Control Oriented Modelling Methodology}

Complex nonlinear models are very useful for off-line operations (for instance, calibration and simulation). Fine mathematical representations such as the SaintVenant equations for describing the open-flow behavior (Mays, 2004) or pressureflow models allow the simulation of those systems with enough accuracy to observe specific phenomena, useful for design and investment planning. However, for on-line computation purposes such as those related to global management, a simpler control-oriented model structure should be conveniently selected. This simplified model includes the following features:

- Representation of the main network dynamics: It must provide an evaluation of the main representative hydrological/hydraulic variables of the network and their response to control actions at the actuators.

- Simplicity, expendability, flexibility and computational speed: It must use the simplest approach capable of achieving the given purposes, allowing very easily to expand and/or modify the modelled portion of the network.

Several modelling techniques dealing with the operational control of water systems have been presented in the literature, see Brdys \& Ulanicki (1994); Mays 
(2004) and the references therein. Here, a control-oriented modelling approach is outlined, which follows the principles presented in Cembrano et al. (2004) and Ocampo-Martinez et al. (2013). The extension to include the pressure-model can be found in the references provided by Brdys \& Ulanicki (1994) and Mays (2004).

A water system generally contains tanks, which store the drinking water that comes from the network sources, a network of pipes and open flow canals, and a number of demands. Valves and/or pumping stations are elements that allow to manipulate the water flow according to a specific policy and to supply water requested by the network users. These flows are chosen by a global management strategy.

The water system model can be considered as composed of a set of constitutive elements, which are presented and discussed below.

\subsection{Tanks and Reservoirs}

Water tanks/reservoirs provide the entire network with the water storage capacity. The mass balance expression relating the stored volume $v$, the manipulated inflows $q_{\mathrm{in}}^{i, j}$ and outflows $q_{\text {out }}^{i, l}$ (including the demand flows as outflows) for the $i$-th tank can be written as the discrete-time difference equation

$$
v_{i}(k+1)=v_{i}(k)+\Delta t\left(\sum_{j} q_{\mathrm{in}}^{i, j}(k)-\sum_{l} q_{\mathrm{out}}^{i, l}(k)\right),
$$

where $\Delta t$ is the sampling time and $k$ denotes the discrete-time instant. The physical constraint related to the range of admissible water in the $i$-th tank is expressed as

$$
\underline{v}_{i} \leq v_{i}(k) \leq \bar{v}_{i}, \quad \text { for all } k,
$$

where $\underline{v}_{i}$ and $\bar{v}_{i}$ denote the minimum and the maximum admissible storage capacity, respectively. As this constraint is physical, it is impossible to send more water 
to a tank than it can store, or draw more water than the stored amount. Although $\underline{v}_{i}$ might correspond to an empty tank, in practice this value can be set as nonzero in order to maintain an emergency stored volume enough to supply for facing extreme circumstances.

For simplicity purposes, the dynamic behavior of these elements is described as a function of the volume. However, in most of the cases, the measured variable is the tank water level (by using level sensors), which implies the computation of the water volume taking into account the tank geometry.

\subsection{Actuators}

Two types of control actuators are considered: valves/gates and pumps (more precisely, complex pumping stations). The manipulated flows through the actuators represent the manipulated variables, denoted as $q_{u}$. Both pumps and valves/gates have lower and upper physical limits, which are taken into account as system constraints. As in (2), they are expressed as

$$
\underline{q}_{i} \leq q_{u_{i}}(k) \leq \overline{q_{u}}, \quad \text { for all } k,
$$

where ${\underline{q_{u}}}_{i}$ and $\overline{q_{u}}$ denote the minimum and the maximum flow capacity, respectively.

\subsection{Nodes}

These elements correspond to the points in the whole water system where water flows are merged or split. Thus, the nodes represent mass balance relations, being modelled as equality constraints related to inflows (from other tanks through valves or pumps) and outflows, the latter being represented not only by manipulated flows but also by demand flows. The expression of the mass conservation in 
these nodes can be written as

$$
\sum_{j} q_{\text {in }}^{i, j}(k)=\sum_{h} q_{\text {out }}^{i, h}(k) .
$$

From now on and with some abuse of notation, node inflows and outflows are still denoted by $q_{\text {in }}$ and $q_{\text {out }}$, respectively, despite the fact that they can be manipulated flows and hence denoted by $q_{u}$, if required.

\subsection{River Reaches}

A single canal reach can be approximated by using the modelling approach proposed by Litrico \& Fromion (2004) that leads to the following relation between the upstream $\left(q_{\text {ups }}\right)$ and downstream $\left(q_{d n s}\right)$ flows:

$$
q_{d n s}(k+1)=a_{1} q_{d n s}(k)+b_{0} q_{u p s}(k-d)
$$

where $d=\tau_{d} / T_{s}, \tau_{d}$ is the downstream transport delay, $T_{s}$ is the sampling time, $b_{0}=1-a_{1}$ and $a_{1}=e^{-\frac{T_{s}}{T}}$.

\subsection{Demand and Irrigation Sectors}

Demand and irrigation sector represents the water demand made by the network users of a certain physical area. It is considered as a measured disturbance of the system at a given time instant. The demand in urban areas can be anticipated by a forecasting algorithm that is integrated within the MPC closed-loop architecture. The demand forecasting algorithm typically uses a two-level scheme composed by $(i)$ a time-series model to represent the daily aggregate flow values, and (ii) a set of different daily flow demand patterns according to the day type to cater for different consumption during the weekends and holidays periods. Every pattern consists of 24 hourly values for each daily pattern (Quevedo et al., 
2010). This algorithm runs in parallel with the MPC algorithm. The daily series of hourly-flow predictions are computed as a product of the daily aggregate flow value and the appropriate hourly demand pattern. On the other hand, irrigation demand is typically planned in advance with farmers. Pre-established flows for irrigation are in the irrigation areas in certain periods of the year.

\section{MPC Modelling for Supply and Transportation Layers}

\subsection{Model Predictive Control}

MPC is one of the most advanced control methodologies which has made a significant impact on industrial control. MPC does not consider a specific control strategy but a very wide range of control methods which make an explicit use of the process model to obtain the control signal by minimizing an objective function which represent the desired control goals. MPC can handle multi-variable control problems and it can consider actuator limitations as well as operational and physical constraints.

The standard MPC problem based on the linear discrete-time prediction model is considered as described in Maciejowski (2002):

$$
\begin{aligned}
x(k+1) & =A x(k)+B u(k), \\
y(k) & =C x(k),
\end{aligned}
$$

where $x(k) \in \mathbb{R}^{n_{x}}$ is the state vector and $u(k) \in \mathbb{R}^{n_{u}}$ is the vector of command variables at time step $k$, and $y(k) \in \mathbb{R}^{n_{y}}$ is the vector of the measured output. Following the formalism provided by Maciejowski (2002) for the basic formulation of a predictive control, the cost function is assumed to be quadratic and the constraints are in the form of linear inequalities. Thus, the following basic optimization problem 
(BOP) has to be solved:

$$
\begin{array}{ll} 
& \min _{\left(u(0 \mid k), \cdots, u\left(H_{p}-1 \mid k\right)\right)} J(k) \\
\text { s.t. } & x(i+1 \mid k)=A x(i \mid k)+B u(i \mid k), \quad i=1, \cdots, H_{p}, \\
& x(0 \mid k)=x_{k}, \\
& x_{\min } \leq x(i \mid k) \leq x_{\max }, \quad i=1, \cdots, H_{p}, \\
& u_{\text {min }} \leq u(i \mid k) \leq u_{\max }, \quad i=0, \cdots, H_{p-1},
\end{array}
$$

As described above $J$ is a performance index, representing the operational goals of the system. And $H_{p}$ is the prediction horizon, $x(0)$ is the initial condition of the state vector, $u_{\min }$ and $u_{\max }$ are known vectors defining the saturation constraints on inputs variables (operational ranges). Problem (7) can be recast as a Quadratic Programming (QP) problem, whose solution:

$$
\mathcal{U}^{*}(k) \triangleq\left[u^{*}(0 \mid k) \cdots u^{*}\left(H_{p}-1 \mid k\right)\right]^{T} \in \mathbb{R}^{H_{p} m \times 1}
$$

is a sequence of optimal control inputs that generates an admissible state sequence. At each sampling time $k$, Problem (7) is solved for the given measured (or estimated) current state $x(k)$. Only the first optimal move $u^{*}(0 \mid k)$ of the optimal sequence $\mathcal{U}^{*}(k)$ is applied to the process:

$$
u_{M P C}(k)=u^{*}(0 \mid k)
$$

the remaining optimal decisions are discarded and the optimization is repeated at time $k+1$.

\subsection{State Space Model for Supply Layer}

The state space model of supply layer has two kinds of states and control variables. First kind of state variable represents reservoirs and the managed variable corresponds to actuator flows: 


$$
x(k+1)=A x(k)+B u(k)+B_{p}[d(k)-\varepsilon(k)], \quad k \in \mathbb{Z}
$$

where

$$
\begin{aligned}
& x(k) \in \mathbb{R}^{n_{x}} \quad \text { state variables represent volumes } \\
& u(k) \in \mathbb{R}^{n_{u}} \quad \text { control corresponds to actuator flows } \\
& d(k) \in \mathbb{R}^{n_{d}} \quad \text { disturbances correspond to demands } \\
& \varepsilon(k) \in \mathbb{R}^{n_{d}} \quad \text { slack variables for unsatisfied demands }
\end{aligned}
$$

At this function, $\varepsilon(k)$ is introduced to control the amount of demand which has not been satisfied.

The second kind of states and control variable represent river flows in a river reach model with delays. For simplicity and brevity of the explanation, consider river reach model (5) as a transport delay (Evans et al., 2011):

$$
q_{\text {out }_{i}}=q_{\text {in }_{i}}\left(k-\tau_{d}\right)
$$

where $\tau_{d}$ represents the delayed value. For time delays associated with flows within the network, the following auxiliary state equations are introduced:

$$
\begin{gathered}
x_{j, 1}(k+1)=q_{j}(k) \\
x_{j, i+1}(k+1)=x_{j, i}(k), i=1, \cdots, \tau_{d}
\end{gathered}
$$

where

$$
\begin{array}{cl}
x_{j, i}(k) \in \mathbb{R}^{n_{x}^{\prime}} & \text { state variables represent flows } \\
q_{j}(k) \in \mathbb{R}^{n_{u}^{\prime}} & \text { flows, part of control variables } \\
\tau_{d} \in \mathbb{Z} & \text { delay }
\end{array}
$$


More details on how this approach can be extended to the case that river reach model (5) is not just considered as a delay can be found in Evans et al. (2011).

After combining (12) and (13) with (10), we have a new augmented state space representation

$$
\widetilde{x}(k+1)=\widetilde{A} \widetilde{x}(k)+\widetilde{B} \widetilde{u}(k)+\widetilde{B}_{p}[d(k)-\varepsilon(k)], \quad k \in \mathbb{Z}
$$

where

$$
\widetilde{x}(k)=\left[\begin{array}{c}
x(k) \\
x_{j},{ }_{i}(k)
\end{array}\right], \quad \widetilde{u}(k)=\left[\begin{array}{c}
u(k) \\
q_{j}(k)
\end{array}\right]
$$

and

$$
\begin{aligned}
& \widetilde{x}(k) \in \mathbb{R}^{\widetilde{n}_{x}} \\
& \widetilde{u}(k) \in \mathbb{R}^{\widetilde{n}_{u}}
\end{aligned}
$$

According to (2) and (3), all the variables are subject to the following inequality constraints:

$$
\begin{aligned}
& \widetilde{x}_{\text {min }} \leq \tilde{x}(k) \leq \widetilde{x}_{\text {max }} \\
& \widetilde{u}_{\text {min }} \leq \widetilde{u}(k) \leq \widetilde{u}_{\text {max }} \\
& \varepsilon_{\text {min }} \leq \varepsilon(k) \leq \varepsilon_{\text {max }}
\end{aligned}
$$

where $\widetilde{x}_{\min }$ and $\widetilde{x}_{\max }$ are physical limitations of the reservoirs, while $\widetilde{u}_{\min }$ and $\widetilde{u}_{\max }$ are physical limitations of the river flows. The range of $\varepsilon_{m i n}$ lies between zero and the related demand.

As described at Section 2, the balance at every node should be satisfied, where $E, E_{d}, E_{\widetilde{x}}$ are matrices which parameters can be obtained from topology of the 
water network:

$$
E \widetilde{u}+E_{d} d-E_{d} \varepsilon+E_{\widetilde{x}} \widetilde{x}=0
$$

During the consumption process, water storage of reservoir should be kept above a given level (named as water safety level) which is used as emergency supply for drought period. Any situation below the emergency level should be penalized using soft constraints:

$$
\begin{gathered}
\widetilde{x} \geq \widetilde{x_{r}}-\varepsilon_{\widetilde{x}} \\
\varepsilon_{\widetilde{x}} \geq 0
\end{gathered}
$$

where $\widetilde{x_{r}}$ is the water safety level and $\varepsilon_{\widetilde{x}}$ is the slack to $\widetilde{x_{r}}$.

Stability of MPC is one important issue that has drawn a lot of attention since local optimization in a finite preview horizon does not guarantee stability in general (Lee et al., 1996). The most widely referenced approach to guarantee stability in MPC procedures is to add an equality constraint on the final state in the prediction horizon (a so called end-state constraint) or put a weight on the final state in the objective function (De Nicolao \& Scattolini, 1998; Genceli \& Nikolaou, 1993; Kwon \& Pearson, 1977; Kwon \& Byun, 1989; Mayne \& Michalska, 1990; Thomas, 1975). Another approach is to use an infinite prediction horizon with a finite control horizon (Rawlings \& Muske, 1993), making it possible to apply standard linear quadratic regulator $(\mathrm{LQR})$ theory to guarantee stability (Kwakernaak \& Sivan, 1972; Cheng \& Krogh, 2001). In this paper, additive constraints on the states about the penalty water level in reservoirs is preferred to using a terminal condition in order to avoid infeasibility of the MPC strategies due to uncertainty in the dynamic model. However, it is important to take into account that using a finite state horizon (e.g. 30 days) when the reservoir memory is considerably 
longer might produce strategies that do not guarantee longer-term stability. This methodological issue will be analyzed in future work.

The state space model of the transportation layer is simpler since the states corresponds to the tank volumes and the manipulated variables are the flows in pumps and valves. This leads to a standard state space representation (6) for the transportation layer. More details can be found in Ocampo-Martinez et al. (2013).

\subsection{Operational Goals}

\subsubsection{Operational Goals for Supply Layer}

The supply network is operated with a 30-day horizon, at daily time interval. The main operational goals to be achieved in the supply network are:

- Operational safety $\left(J_{\text {safety }}\right)$ : This criterion refers to maintain appropriate water storage levels in dams and reservoirs for emergency-handling. Operated in both supply and transportation layers.

- Demand management $\left(J_{\text {demand }}\right)$ : This is especially important in the supply layer when urban and irrigation demands exist since urban demands must be fully satisfied while irrigation demands allow some degree of slackness.

- Balance management $\left(J_{\text {balance }}\right)$ : This is operated only at supply layer which is necessary for keeping rivers or reservoirs consumed in a balanced way and escaping water deficit problem for both of the two rivers in a longer time.

- Minimizing waste $\left(J_{\text {mwaste }}\right)$ : Take into account that the river water eventually goes to the sea, this term gets to avoid unnecessary water release from 
reservoirs (release water that does not meet any demand and is eventually wasted).

- Environment conservation ( $\left.J_{\text {ecological }}\right)$ : Water sources such as boreholes, reservoirs and rivers are usually subject to operational constraints to maintain water levels and ecological flows.

Above mentioned goals lead to the following function:

$$
\begin{aligned}
J & =J_{\text {safety }}+J_{\text {demand }}+J_{\text {mwaste }}+J_{\text {balance }} \\
& =\varepsilon_{\widetilde{x}}(k)^{\top} W_{\widetilde{x}} \varepsilon_{\widetilde{x}}(k)+\varepsilon(k)^{\top} W_{f} \varepsilon(k) \\
& +\left(\widetilde{u}_{i \ldots j}(k)-\widetilde{u}_{s}(k)\right)^{\top} W_{\widetilde{w}}\left(\widetilde{u}_{i \ldots j}(k)-\widetilde{u}_{s}(k)\right) \\
& +\left(\left(\begin{array}{lllllll}
0 & \ldots 0 & \frac{1}{x i_{\text {max }}^{\prime}} & 0 \ldots 0 & \frac{-1}{x j_{\text {max }}^{\prime}} & 0 \ldots 0
\end{array}\right) \widetilde{x}(k)\right)^{\top} w_{\widetilde{m}} \\
& \times\left(\left(\begin{array}{lllllll}
0 & \ldots 0 & \frac{1}{x i_{\text {max }}^{\prime}} & 0 \ldots 0 & \frac{-1}{x j_{\text {max }}^{\prime}} & 0 \ldots 0
\end{array}\right) \widetilde{x}(k)\right)
\end{aligned}
$$

where

$$
\begin{aligned}
\varepsilon_{\widetilde{x}}(k) & =\widetilde{x}(k)-\widetilde{x}_{r} \\
\widetilde{u} & =\Theta \Delta \widetilde{u}+\Pi \widetilde{u}(k-1) \\
\Delta \widetilde{u}(k) & =\widetilde{u}(k)-\widetilde{u}(k-1)
\end{aligned}
$$

and:

$$
\Theta=\left(\begin{array}{cccc}
I_{m_{i}} & 0 & \ldots & 0 \\
I_{m_{i}} & I_{m_{i}} & \ldots & 0 \\
\vdots & \vdots & \ddots & \vdots \\
I_{m_{i}} & I_{m_{i}} & \ldots & I_{m_{i}}
\end{array}\right), \quad \Pi=\left(\begin{array}{c}
I_{m_{i}} \\
I_{m_{i}} \\
\vdots \\
I_{m_{i}}
\end{array}\right) .
$$

and $W_{\widetilde{x}}, W_{f}, W_{\widetilde{w}}, W_{\widetilde{x}}, w_{\widetilde{m}}$ are the related weights which decide the priorities (established by the water network authorities) for all the terms appearing in the objective 
function. The weight tuning method proposed in Toro et al. (2011), based on computing the Pareto front of the multi-objective optimization problem presented in (20), is used in this paper. The initial step of this tuning approach is to find what are known as the anchor points that correspond to the best possible value for each objective obtained by optimizing a single criterion at a time. Then, a normalization procedure is applied, a Management Point (MP) defined by establishing objective priorities is defined, and the optimal weights are determined by computing those that minimize the distance from the solutions of the Pareto front and the MP.

It should be noticed that the term $J_{\text {safety }}$ in (20) contains the ecological flows, implicitly including $J_{\text {ecological }}$. The reason is that flows in the rivers are modelled as additional state variables as discussed before. Variables $\widetilde{u}_{i \ldots j}(k)$ are the flows from the rivers to the sea. $\widetilde{u}_{s}(k)$ are their ecological penalty levels. $x_{i}$ and $x_{j}$ are two main reservoirs located in two different rivers.

\subsubsection{Operational Goals for Transportation Layer}

The transportation network is operated with a 24-hour horizon, at hourly time interval. The main operational goals to be achieved in the transportation network are:

- Cost reduction $\left(J_{\text {cost }}\right)$ : Water cost is usually related to acquisition, which may have different prices at different sources and elevations, affected by power tariffs which may vary during a day.

- Operational safety $\left(J_{\text {safety }}\right)$ : This criterion refers to maintain appropriate water storage levels in dams and reservoirs of the network for emergencyhandling. 
- Control actions smoothness ( $\left.J_{\text {smoothness }}\right)$ : The operation of water treatment plants and main valves usually requires smooth flow set-point variations for best process operation.

Above mentioned goals lead to the following function:

$$
\begin{aligned}
J & =J_{\text {safety }}+J_{\text {smoothness }}+J_{\text {cost }} \\
& =\varepsilon_{\widetilde{x}}(k)^{\top} W_{\widetilde{x}} \varepsilon_{\widetilde{x}}(k)+\Delta \widetilde{u}(k)^{\top} W_{\widetilde{u}} \Delta \widetilde{u}(k) \\
& +W_{a}\left(a_{1}+a_{2}(k)\right) \widetilde{u}(k)
\end{aligned}
$$

where

$$
\begin{aligned}
\varepsilon_{\widetilde{x}}(k) & =\widetilde{x}(k)-\widetilde{x}_{r} \\
\widetilde{u} & =\Theta \Delta \widetilde{u}+\Pi \widetilde{u}(k-1) \\
\Delta \widetilde{u}(k) & =\widetilde{u}(k)-\widetilde{u}(k-1)
\end{aligned}
$$

and $W_{\widetilde{x}}, W_{\widetilde{u}}, W_{a}$ are the related weights.

The vectors $a_{1}$ and $a_{2}$ contain the cost of water treatment and pumping, respectively.

\subsection{Formulation of the optimization problem}

The objective function (20) and (21) of the MPC problem can be formulated in the following way:

$$
J=z^{T} \Phi z+\phi^{T} z+c
$$

where

$$
z=\left[\begin{array}{lll}
\Delta \widetilde{u} & \varepsilon_{\widetilde{x}} & \varepsilon
\end{array}\right]^{T}
$$

and $c$ is a constant value produced by vector calculation. 
This allows to determine the optimal control actions at each instant $k$ by solving a quadratic optimization problem by means of quadratic programming (QP) algorithm in the form:

$$
\begin{gathered}
\min _{z} z^{\top} \Phi x+\phi^{\top} z \\
A_{1} z \leq b_{1} \\
A_{2} z=b_{2}
\end{gathered}
$$

\section{Temporal Multi-layer MPC Scheme}

\subsection{Multi-layer Model Predictive Control}

There are three basic methods of decomposition of the overall control objective (Brdys \& Ulanicki, 1994):

- temporal hierarchy

- spatial hierarchy

- functional hierarchy

Among them, temporal hierarchy is particularly important in the control of water systems and it will be presented in the following sections (Brdys \& Tatjewski, 2005).

The general principle of temporal multi-layer MPC is that decision of a higher layer have a wider temporal extent than the one of a lower layer. At the same time, because of the limited capacity, the higher level decision units process more aggregated information than the lower ones. In this paper, a two-level structure related to the supply and transportation layers of a water network is proposed as shown in Fig. 1. 


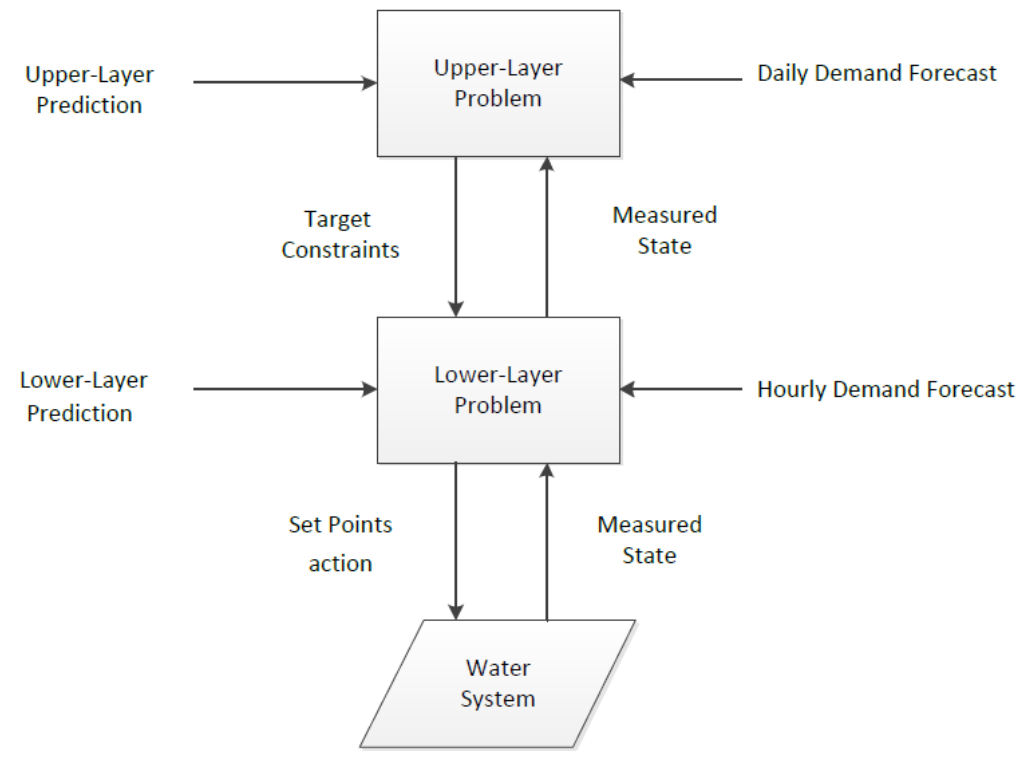

Figure 1: Temporal hierarchy Multi-layer MPC

The systems correspond to these two layers and operated according to different goals and time scales. However, both layers use MPC to compute control strategies and controls can be characterized by the pair $\left(H_{p}, T_{s}\right)$, where $H_{p}$ is a the time horizon for the optimization problem, $T_{s}$ is the sampling time.

The operation of the hierarchical structure is presented by Algorithm 1 where the pair $(k, m)$ is used to fix a point on a time scale, with the following meaning: $k$ means the current day, $m$ means the current hour within the current day. $K$ will denote the number of days over which the scheme is in operation and $M$ (which is equal to 24) will be the number of hours in a day. For convenience the following notation is chosen: $x(k)=x(k, m)$, and $x^{*}$ to denote the state of the physical system. 


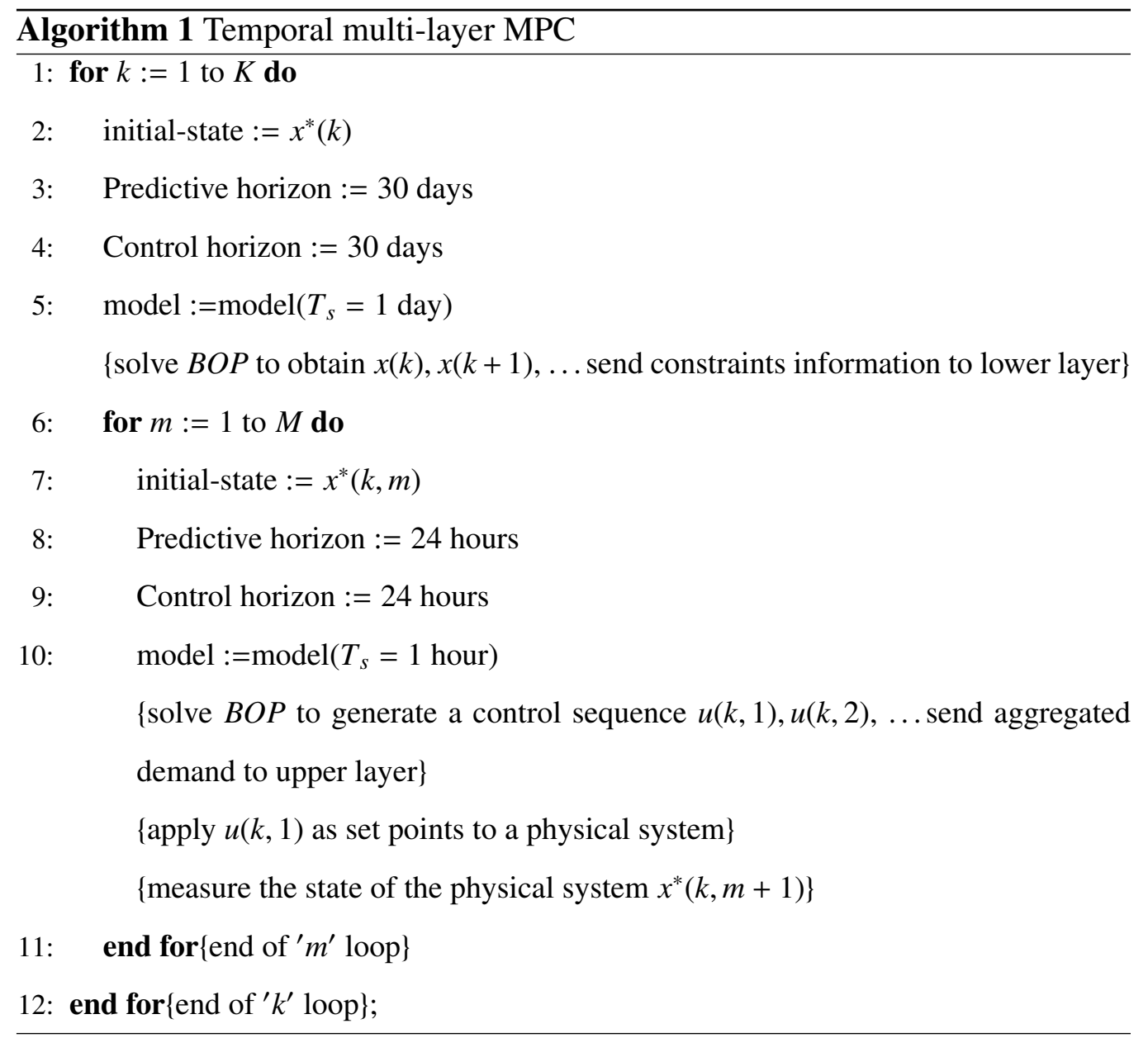




\subsection{Temporal Multi-layer Coordination Techniques}

As shown in Fig. 1, the way to represent interaction between the upper (daily model for the supply layer) and lower (hourly model for the transportation layer) layers relies on two elements:

- Measured disturbance $\left(M_{s}\right)$ : which handles the related aggregated demands at the transportation layer every predictive horizon hours as communication information to the supply layer.

- Target constraint $\left(T_{d}\right)$ : which expresses management policies at the supply layer to the transportation layer in the form of control constraints.

\subsubsection{Measured Disturbance}

In the topology of the supply layer, the whole transportation layer is simplified as one aggregated demand. Measured state in every optimization process for supply layer should be sum of the related demand every prediction horizon (here is 24 hours)

$$
M_{s}(k)=\sum_{m=1}^{24} d_{t}(k, m)
$$

where $d_{t}(k, i)$ is demand vector at the transportation layer corresponding to the $k$-th day.

Thus, $M_{s}(k)$ should be considered as the demand for the supply layer

$$
d_{s}(k)=M_{s}(k)
$$

\subsubsection{Target Constraints}

The goal for the temporal coordination algorithm is transferring management policies from the upper (supply) to the lower (transportation) layer. In order to 
achieve this coordination, the following constraint is added to the the lower layer MPC:

$$
\sum_{m=1}^{24} u(k, m) \leq T_{d}(k)
$$

where $u$ is the shared control vector between supply and transportation layers.

This constraint is introduced in order to enforce that the amount of water decided to be transferred from the supply to the transportation layer by the upper layer MPC is respected by the lower layer MPC. Without such a constraint, the lower layer MPC would decide the amount of water ignoring the upper layer MPC policy.

The coordination working structure is shown at Fig. 2:

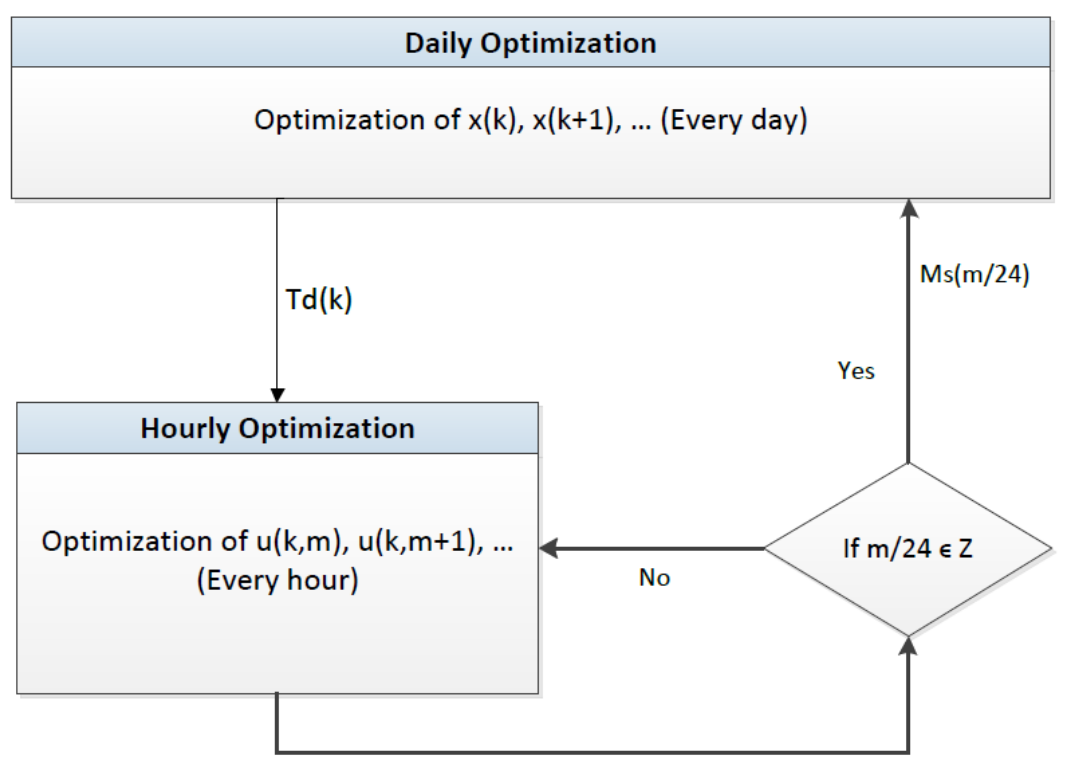

Figure 2: Upper and Lower layer optimizations of multi-layer MPC 


\section{Formulation of the Temporal Multi-layer MPC Scheme}

\subsection{Formulation of Temporal Coordination Problem}

As explained in Section 3, the goal for the temporal coordination algorithm is transferring management policies from the upper (supply) to the lower (transportation) layer. In order to achieve this coordination, the constraint (26) is added to the the lower layer MPC. Algorithm 2 shows how this constraint, that establishes a daily limitation, is generated and adapted at every time iteration of the lower layer MPC that operates at a hourly scale. Algorithm 2 takes into account the following facts when generating the constraint (26).

- after the application of $n$ hourly control actions $u_{s}(m)$ corresponding to the $k$-th day, the total remaining water for this day will be: $T_{d}(k)-\sum_{m=1}^{n} u(m)$

- when limiting the control actions in the prediction horizon $L$, there is a part of control actions $u(m)$ that corresponds to hours of the current day $k$ that should be limited by $T_{d}(k)$, while the control actions correspond to hours of the next day $k+1$ that should be limited by $T_{d}(k)-\sum_{m=1}^{n} u(m)$.

- the generated constraints are added as additional constraints of the BOP problem associated to the lower layer MPC.

\subsection{Formulation for Predicting the Water Demand}

In order to implement the temporal Multi-level MPC approach, two demand forecasts algorithms are needed (see Figure 1). One, at the daily level and the other at the hourly level. These two algorithms are based in the approach proposed by Quevedo et al. (2010): 


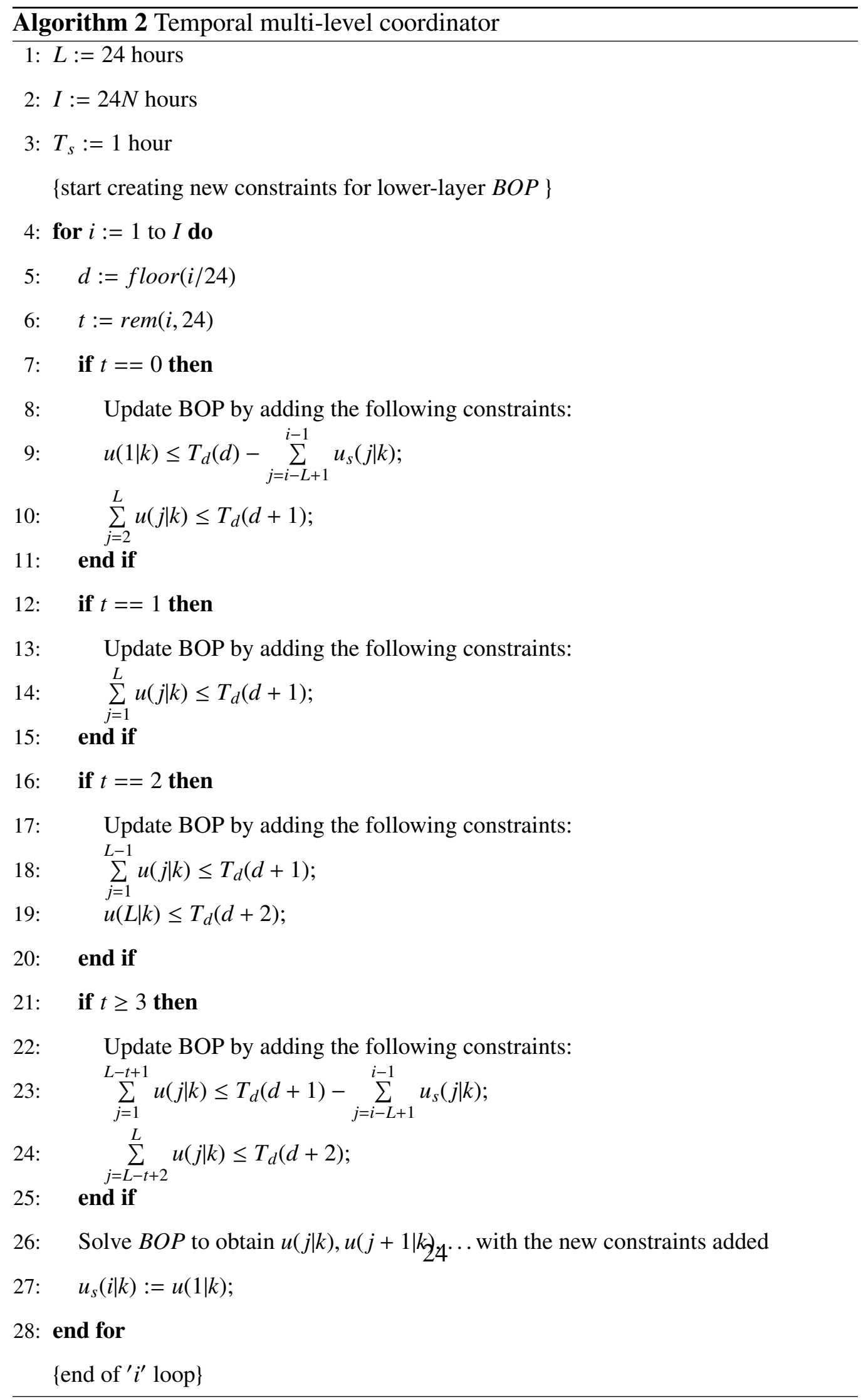


- A time-series modelling to represent the daily demand forecast.

- A set of different daily flow demand patterns according to the day type to cater for different consumption during the weekends and holidays periods. Every pattern consists of 24 hourly values for each daily pattern (hourly demand forecast).

This algorithm will run in parallel with the MPC algorithms both in supply and transportation layers to obtain the pattern of daily and hourly flow demand.

\subsection{Daily demand forecast}

The daily flow model is built on the basis of a time series modelling approach using an ARIMA strategy. A time series analysis was carried out on several daily aggregate series, which consistently showed a weekly seasonality, as well as the presence of deterministic periodic components. A general expression for the daily flow model, to be used for a number of demands in different locations, was derived using three main components:

- A weekly-period oscillating signal, with zero average value to cater for cyclic deterministic behavior, implemented using a second-order (two-parameter) model with two oscillating modes $\left(p_{1,2}=\cos (2 \pi / 7) \pm j \sin (2 \pi / 7)\right)$

$$
\Delta y_{o s c}(k)=\Delta y_{\text {int }}-2 \cos (2 \pi / 7) \Delta y_{\text {int }}(k-1)+\Delta y_{\text {int }}(k-2)
$$

- An integrator takes into account possible trends and the non-zero mean value of the flow data

$$
\Delta y_{\text {int }}(k)=y(k)-y(k-1)
$$


- An autoregressive component to consider the influence of previous flow values within a week. For the general case, the influence of four previous days is considered (28). However, after parameter estimation and significance analysis, the models are usually reduced implementing a smaller number of parameters:

$$
y(k)=-a_{1} y(k-1)-a_{2} y(k-2)-a_{3} y(k-3)-a_{4} y(k-4)
$$

Combining the previous components in the following way the structure of aggregate daily flow model for each demand sensor is therefore:

$$
\begin{aligned}
y_{p}(k)= & -b_{1} y(k-1)-b_{2} y(k-2)-b_{3} y(k-3)-b_{4} y(k-4) \\
& -b_{5} y(k-5)-b_{6} y(k-6)-b_{7} y(k-7)
\end{aligned}
$$

The parameters $b_{1}, \ldots, b_{7}$ should be adjusted using least-squares-based parameter estimation methods and historical data.

\subsection{Hourly demand forecast}

The 1-hour flow model is based on distributing the daily flow prediction provided by the time-series model described in previous section using a one-hourflow pattern that takes into account the daily/monthly variation in the following way:

$$
y_{p h}(k+i)=\frac{y_{p a t}(k, i)}{\sum_{j=1}^{24} y_{p a t}(k, j)} y_{p}(k), \quad i=1, \ldots, 24
$$

where $y_{p}(k)$ is the predicted flow for the current day $k$ using (29) and $y_{p a t}$ is the prediction provided by the flow pattern with the flow pattern class day/month of 
the current day. Demand patterns are obtained from statistical analysis (for more details see Quevedo et al. (2010)).

\subsection{Handling uncertainty}

The main source of uncertainty is related to demands, although some uncertainty in the network dynamics is present as well because the use of simplified control oriented models. In this paper, the proposed MPC controller does not handle the uncertainty explicitly. However, because MPC approach relies on the receding horizon principle, that is based on replanning the control strategy at every iteration, taking into account the measurements collected in real-time from telemetry system, uncertainty will be compensated for to a certain extent. To explicitly address the effect of uncertainty in the MPC controller design, robust MPC approaches may be used. These, in general require representation of uncertainty that may be either deterministic (Chisci et al., 2001; Goulart et al., 2006; Lee \& Yu, 1997; Mayne et al., 2000; Rakovic et al., 2012) or stochastic (G.Calafiore \& F.Dabbene, 2006; Shapiro et al., 2009; Charnes \& Cooper, 1962). The application of the those techniques is let as future research in this paper, since the contribution is mainly concentrated in the coordination between MPC controllers operating at different time scales in a regional water network.

\section{Case Study: Catalunya Regional Water Network}

\subsection{Description}

The Catalunya Regional Water Network lies within the Catalunya Inland Basins, from which the Metropolitan area of Barcelona is fed and where most of the population is concentrated (approximately 5.5 million people). The Catalunya Regional Water Network composed mainly by two rivers (Llobregat and Ter) and 
related components. An assessment based on data obtained by the supply companies in the Barcelona metropolitan area shows that in 2007, 81 percent of the water input came from surface sources. Of the total water input, $90 \mathrm{hm}^{3}$ came from the Llobregat system and $124 \mathrm{hm}^{3}$ from the Ter system. The water flow supplied by the Ter and Llobregat rivers are regulated respectively by three and two reservoirs and purified by one and two water treatment plants, respectively.

In Figure 3, an aggregate model of Catalunya Regional Water Network is provided. According to the definition of functional decomposition, the Catalunya Regional Water Network can be separated into three layers. The supply layer, is composed by rivers Llobregat, Ter and all the connected elements, at the two side of Figure 3. The transportation layer, composed by metropolitan areas and also treatment, desalination plants inside them, is in the center of Figure 3. Demand areas at the transportation layer are represented the distribution layer, which is not described in this network. The hydrological regime of Catalunya, is characterized by the irregularity of its rainfall pattern, which, as is typical of the Mediterranean climate, varies greatly between years. This makes the region especially vulnerable to drought episodes, which are expected to increase due to climate change.

Besides that, according to the historical evolution of water reserves evolution in Llobregat and Ter reservoirs, which are the most important reservoirs in the Catalunya Regional Water Network, in the past thirty years (1982-2012), both reservoirs have had more than 6 times water warning problems, what is worse, in the recent 20 years (1992-2012), the frequency is increasing, as Figure 4 shows. In order to solve this water shortage problem, a desalination plant has been built, which is useful to mitigate the water scarcity, the water comes at a large economic and environmental cost could be quite a big expenditure. So, searching for an 


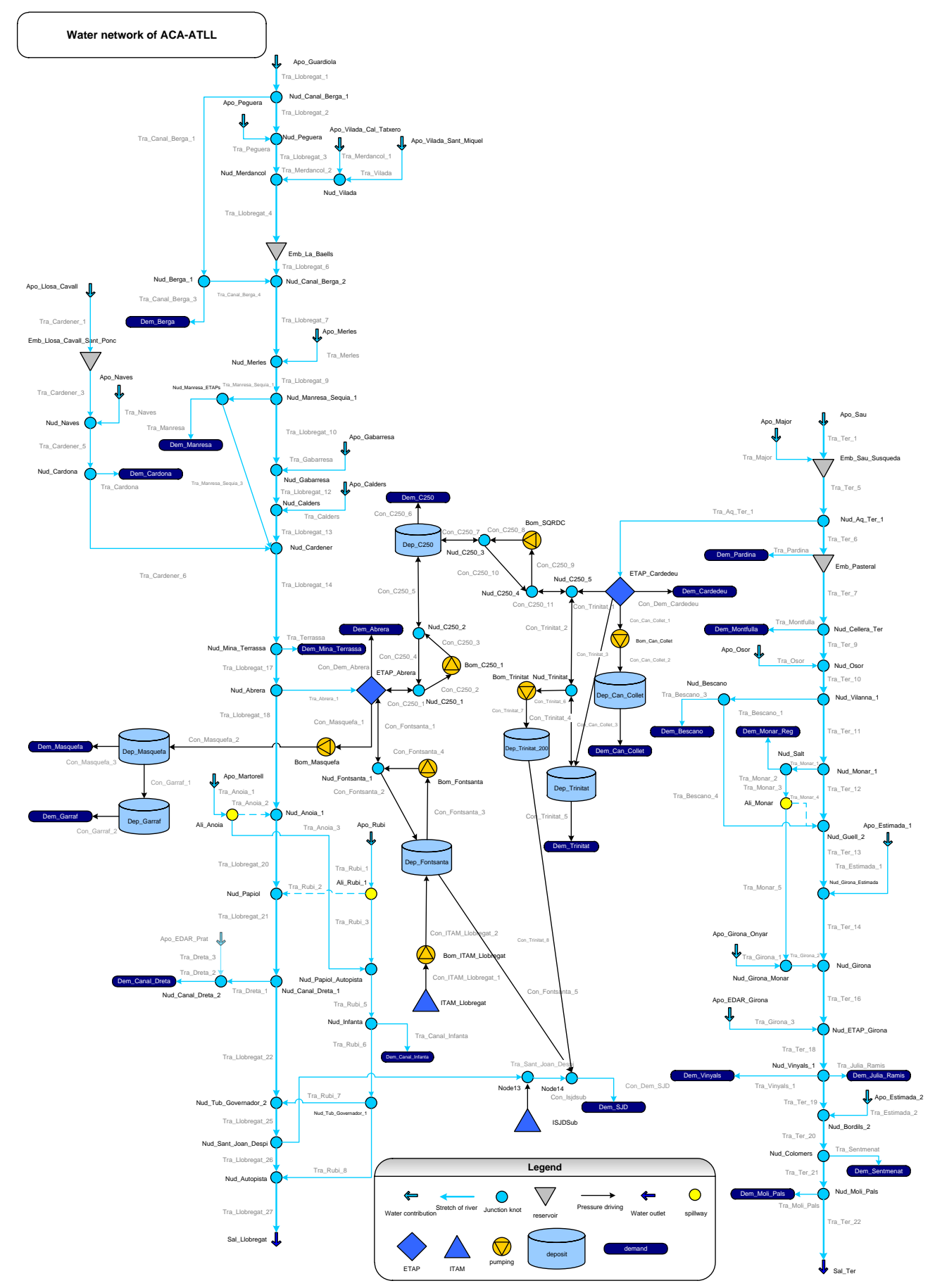

Figure 3: Aggregate diagram of ${ }^{2}$ Catalunya Regional Water Network 
optimal control technique to meet more efficient use of water resources is quite crucial in such a network. This is the motivation for developing the multi-layer MPC scheme proposed in this paper.

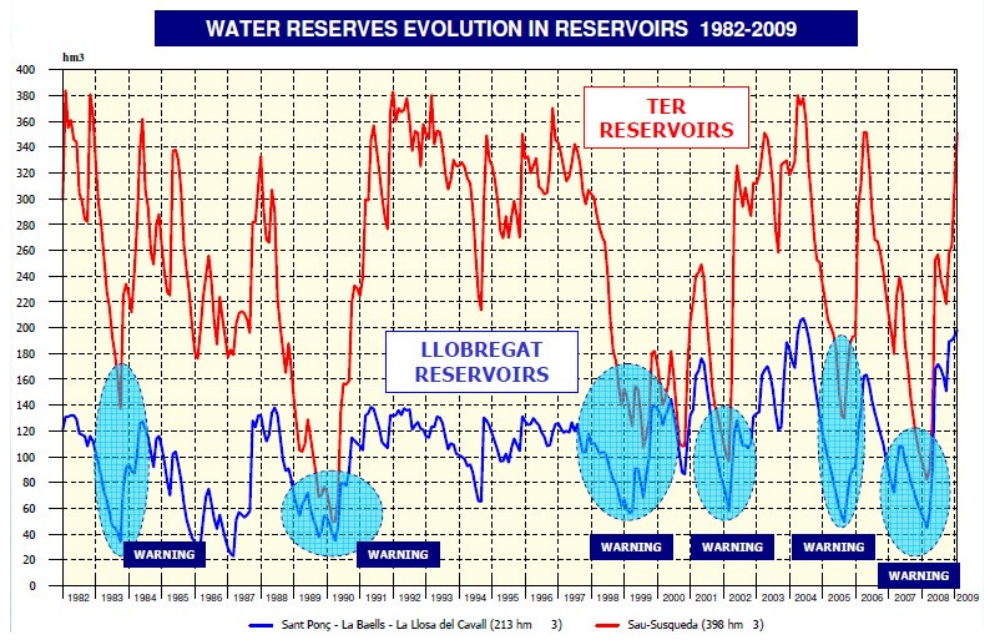

Figure 4: Urgency problem of Catalunya Regional Water Network

\section{Results}

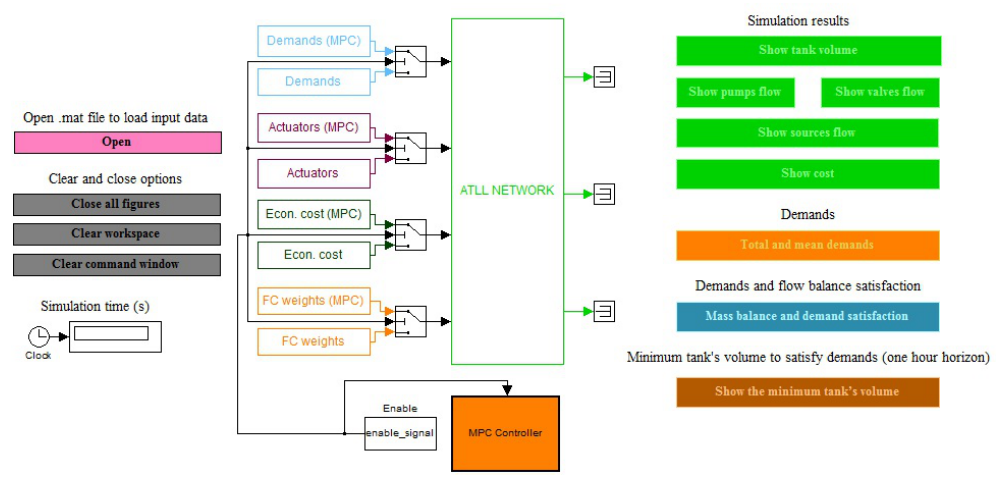

Figure 5: Simulation of the multi-layer MPC control using MATLAB/SIMULINK 
The multi-layer MPC controller presented in Section 3 and Section 4 has been implemented using MATLAB and QP solver of TOMLAB. To test the MPC controller in simulation, a simulator of the Catalunya regional network has been developed (see Figure 5).

\subsection{Supply Layer}

There are three scenarios according to amount of water in different rivers, which are:

- Scenarios 1: More initial water in Llobregat than in Ter.

- Scenarios 2: More initial water in Ter than in Llobregat.

- Scenarios 3: Initial water in both rivers are similar.

According to reality use, for the first two scenarios, when water in one river is adequate while in another river not, management policies will be set to ask water from only one of the rivers. For the scenario 3, when water is similar in both of rivers, according to the balance management, which is one of control objectives in the supply layer, water consumption in both of the rivers will be proportional to their supplying capacity. Table 1 provide detailed results and also improvement of water usages in the two rivers achieved by this proposed multi-layer MPC scheme. In this table, Source means outside sources flow into rivers, Fixed Demand means fixed demands which can not choose water source while Variable Demand is the demand which can receive water from more than one river. $B D$, abbreviation of Balanced Demand, is water volume that has been consumed from each of the reservoirs and PB, abbreviation of Proportion of Balanced demand, is the proportion of $B D$ for the two reservoirs. PR, abbreviation of Proportion of Reservoir 
capacity, is the proportion of storage capacities of the two reservoirs. The similar values for $P B$ and $P R$ is what the multi-layer scheme wants to reach. And $S A$, abbreviation of Supplying Ability, is water supply ability in days of the whole water network before meeting deficit problem at the hypothesis with no rain and no water flow in from outside. The comparisons prove that, after using this proposed MPC scheme, the proportion of water usage from two rivers $(58.93 \%$, which is ratio of Llobregat/Ter) is much closer with proportion of their storage capacities (53.48\%). And what is more, the Catalunya Regional Water Network can supply water 65 days longer than that without balance management, which is a good benefits at the sustainable usage of water resource in the long term perspective.

Table 1: Balancing comparison of Scenarios 1

\begin{tabular}{llllllll}
\hline Sc. & \multicolumn{7}{c}{ Multi-layer MPC Control Scheme } \\
\hline Es. & Source & Fixed Demand & Variable Demand & BD & PB & PR & SA \\
\hline L. & 3008 & 2981 & 724 & 697 & $58.93 \%$ & $53.48 \%$ & 242 Days \\
T. & 3532 & 3518 & 1196 & 1182 & & & \\
\hline Sc. & \multicolumn{7}{c}{ Model Predictive Control } \\
\hline Es. & Source & Fixed Demand & Variable Demand & BD & PB & PR & SA \\
\hline L. & 3008 & 2981 & 7.6 & -19.4 & $-1.02 \%$ & $53.48 \%$ & 177 Days \\
T. & 3532 & 3518 & 1914 & 1900 & & & \\
\hline
\end{tabular}

Figure 7 is one of the examples of one river reach. The plot shows that, after ecological control, water flow at this reach could meet the ecological objective during the whole optimization process.

\subsection{Transportation Layer}

In the transportation layer, as show in Figure 7, water transportation produces cost when pumping water from lower elevation to the higher elevation. In order 


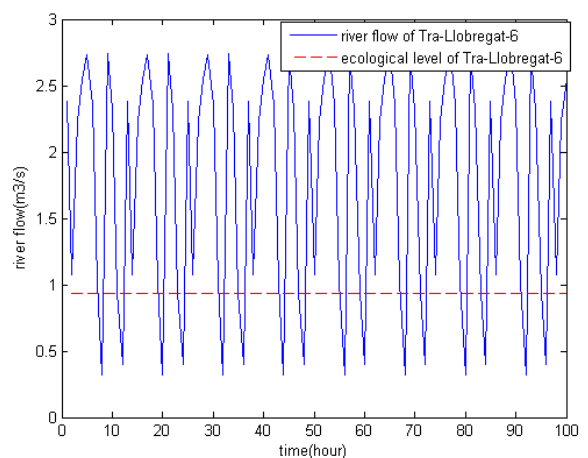

(a) before

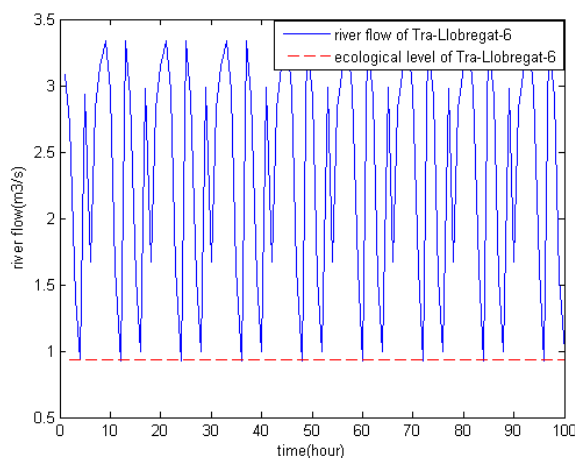

(b) after

Figure 6: River flow comparing with ecological level before and after ecological control in river Llobregat

to show how electrical cost optimization works, the case of Masquefa reservoir, which is marked using a box in the transportation layer will be used as an illustrative example. The figure shows that this reservoir is fed by a pump and supplies water to an urban demand corresponding to the city of Masquefa near Barcelona. Figure 8 shows in the same plot the pump flow and the electricity fee. From this figure, it can be noticed that the pump send more water to the reservoir at the lower price period and less or no water at the higher price period. Figure 9 shows in the same way the water level in the Masquefa reservoir with electricity fee of the pump connected with that reservoir. The water level increases when the connected pump is working corresponding with the night period when the demand is minimal and electricity is cheaper. On the other hand, during the day the level decreases because consumers start demanding water and pumping is minimized because electricity is expensive. The volume of water that should be stored in the reservoir is determined by the MPC controller taking into account of a 24-hour 
ahead demand forecast.

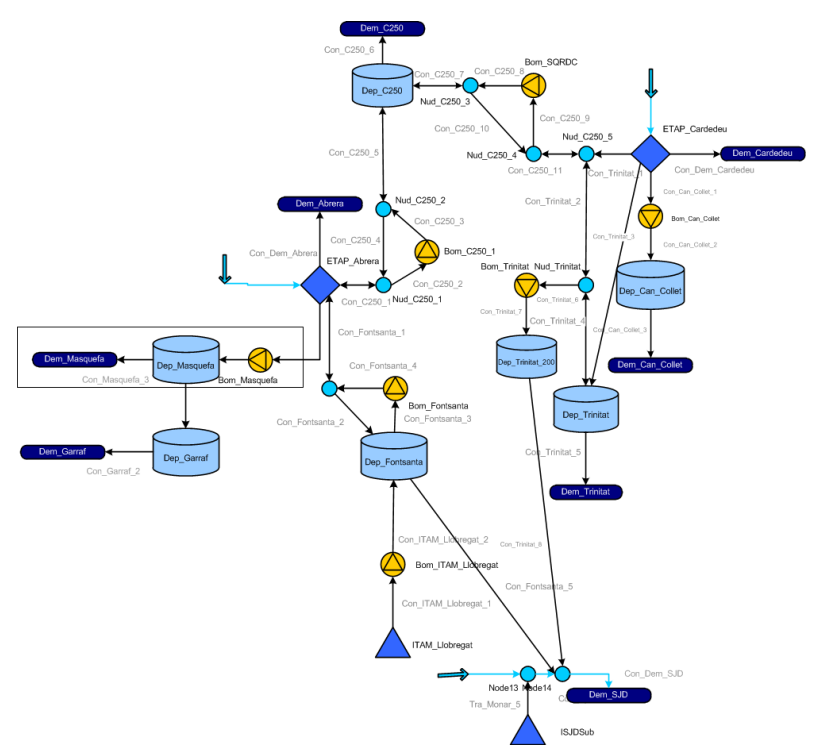

Figure 7: Transportation network

For the rest of the control objectives in transportation layer, Figure 10 shows water level of one tank Dep $_{T}$ rinitat comparing with its safety level before and after the safety level control.

\subsection{Coordination}

During the coordination process, management policies at the supply layer are transferred to the transportation layer using the way of set-point. Figure 11 and Figure 12 show the amount of water consumed by the transportation layer from different rivers for satisfying the same demands before and after coordination, respectively. The two figures prove that average levels of water consumptions from two rivers are much closer after balance management.

Table 2 provides detailed numerical results and compares the obtained control results in terms of economical performance over four days among the three 


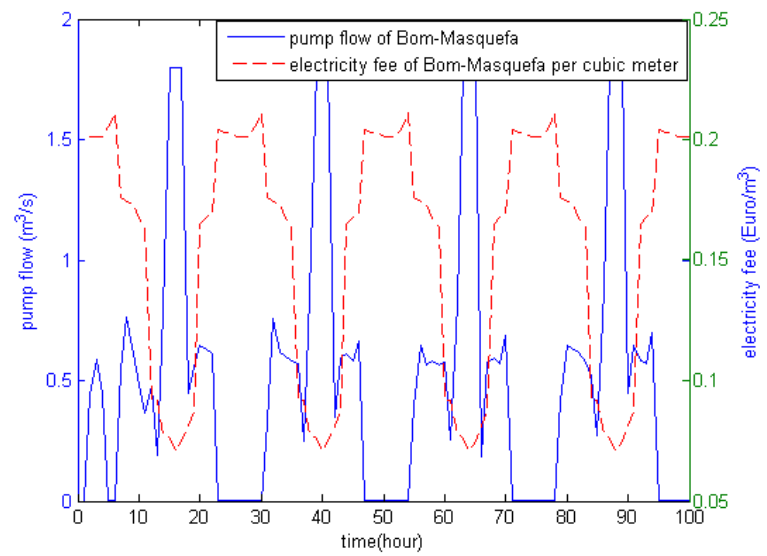

Figure 8: Pump flow with electricity price

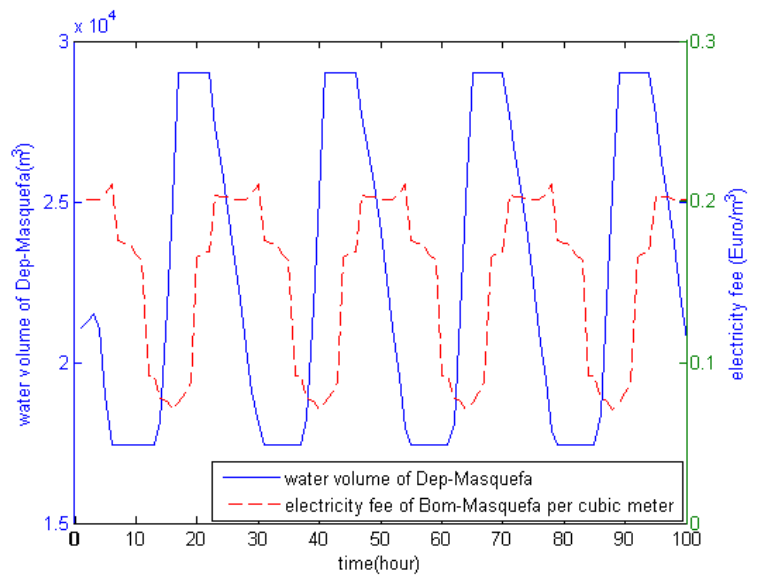

Figure 9: Water level of Dep - Masqueta 


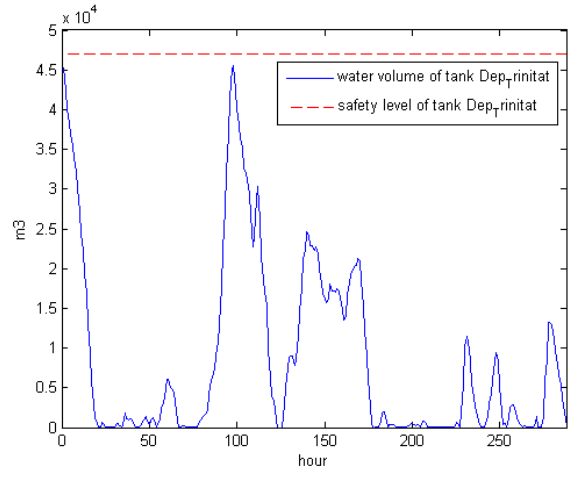

(a) before

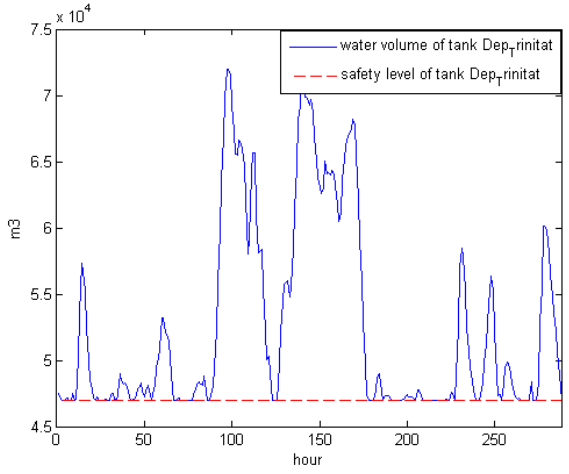

(b) after

Figure 10: Water level of tank Dep $p_{T}$ rinitat before and after safety control starts from the date of $01 / 08 / 2011$

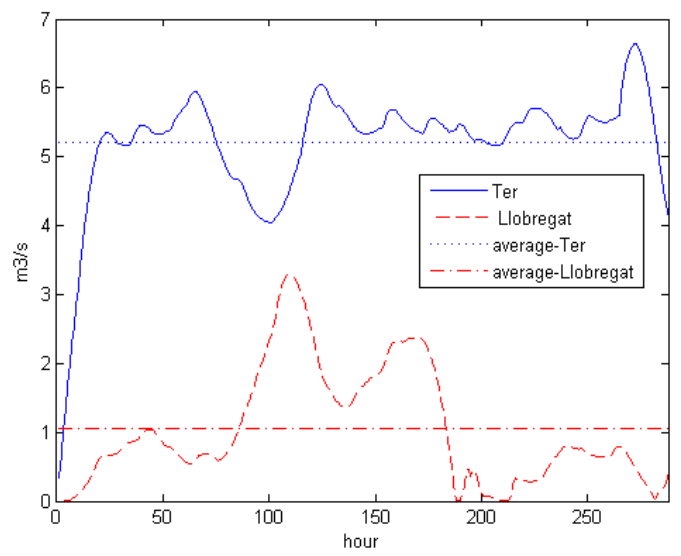

Figure 11: Flows from the two rivers before using temporal coordination with $\mathrm{x}$-time and $\mathrm{y}$-flow axis 


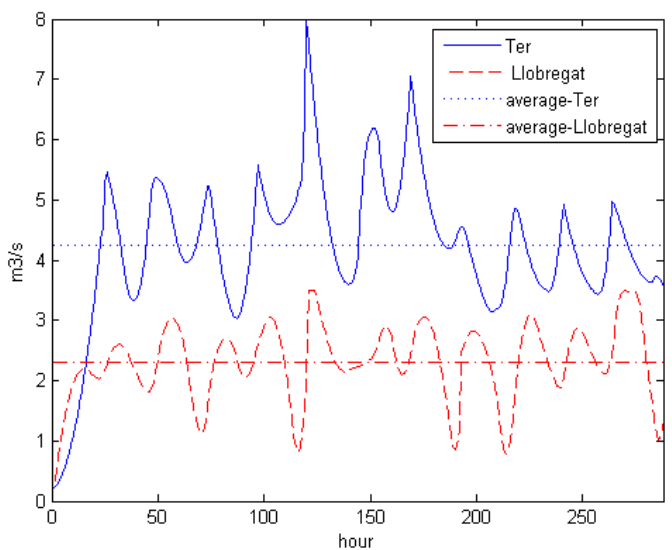

Figure 12: Flows from two rivers after using temporal coordination with $\mathrm{x}$-time and $\mathrm{y}$-flow axis

different control techniques:

\section{- Current Control:}

Control the transportation layer of Catalunya Regional Water Network using heuristic strategies by human operators.

- Multi-layer Model Predictive Control Scheme:

Control the same network using Multi-layer Model Predictive Control techniques with temporal multi-level coordination between the supply and transportation layers.

- Model Predictive Control:

Control the transportation layer of Catalunya Regional Water Network using Model Predictive Control techniques, where no coordination and communication between the supply and transportation layers is used. 
Table 2: Closed-loop performance results (all values in e.u.)

\begin{tabular}{cccccccccc}
\hline \multirow{2}{*}{$\begin{array}{c}\text { Define } \\
\text { Day }\end{array}$} & \multicolumn{3}{c}{ Current Control } & \multicolumn{3}{c}{ Multi-layer MPC } & \multicolumn{3}{c}{ MPC } \\
& Wat. & Ele. & Tot. & Wat. & Ele. & Tot. & Wat. & Ele. & Tot. \\
\hline $11 / 08 / 02$ & 240 & 100 & 340 & 213 & 44 & 257 & 141 & 40 & 181 \\
$11 / 08 / 03$ & 239 & 106 & 345 & 237 & 47 & 284 & 170 & 39 & 209 \\
$11 / 08 / 04$ & 246 & 94 & 340 & 238 & 48 & 286 & 171 & 41 & 212 \\
$11 / 08 / 05$ & 264 & 110 & 374 & 253 & 66 & 319 & 168 & 42 & 210 \\
\hline Proportion & & & & $-5 \%$ & $-50 \%$ & $-18 \%$ & $-34 \%$ & $-61 \%$ & $-42 \%$ \\
\hline
\end{tabular}

In the Table 2, Wat., abbreviation of Water, means water cost during the day, while Ele., abbreviation of Electricity, shows electricity cost and Tot., abbreviation of Total, means the total cost which include both water and electricity. The indices representing costs are given in economic units (e.u.) instead of Euro due to confidentiality restrictions. The row of Proportion is the improved proportion to the current control. From this table, the result shows that, Multi-layer MPC technique with temporal coordination is better than the current control but a little worse than MPC technique without coordination at the point of economical cost, especially of water source cost. The explanation is that while introducing coordination techniques, management policies at the supply layer have also been introduced to the transportation layer. As a consequence, it could happen that demands at the transportation layer have to consume less water from the cheaper river while consume more from the other river which increases the cost. From the perspective of long term, sustainable usage and ecological protection of rivers have been achieved at the price of certain limited cost. Besides that, even from the economical perspective, the Multi-layer MPC with coordination techniques is more feasible than MPC without coordination because the multi-layer MPC can 
make the Catalunya Regional Water Network supply water for 65 days longer as Table 1 shows, which can save much economical expense for solving deficit problem.

\section{Conclusions and Future Work}

In this paper, a multi-layer MPC scheme with multi-level coordination for regional water supply systems is proposed. The need of multi-layer scheme derives from the fact that different networks in the water supply and transportation systems are operated according to different management goals, with different time horizon. While the management of the supply network is mainly concerned with long term safe-yield and ecological issues, the transportation layer must achieve economic goals in the short term (hourly strategy), while meeting demands and operational constraints. The use of the multi-layer modelling and the temporal hierarchy MPC coordination techniques proposed in this paper makes it possible to realize communication and coordination between the two layers in order to let individual operational goals affect to each other, and finally, obtain short-term strategies which can effectively consider long-term objectives as well.

According to objective functions, multi-layer MPC is used to generate control strategies for the complete regional water system to meet as much as demand using optimized economical cost, safety water level in reservoirs, ecological flows in rivers and smooth flow control in actuators. The case study of the Catalunya Regional Water Network has been used to exemplify and verify the proposed management methodology. Results have shown the effectiveness of the proposed modelling and control methodologies allowing to establish a trade-off between short and long-term goals altogether that would not be possible if separate controls were 
applied. This is the main achievement of the proposed scheme.

Uncertainty is handled in an implicit way by the MPC approach by means of the receding horizon philosophy that replans the control strategy after each iteration considering the measurements provided by the water network telemetry system. The explicit handling of uncertainties may be addressed using robust MPC techniques, and this will be addressed as future research.

\section{Acknowledgements}

The authors thank ACA, ATLL and ADASA for providing the case study as well as for sharing their hydrological management expertise. This research has been partially funded by CDTI (MCyT) project HIDROPTIM IDI-20100722, the DGR of Generalitat de Catalunya (SAC group Ref. 2009/SGR/1491), the AGAUR by an FI grant and by EFFINET grant FP7-ICT-2012-318556 of the European Commission.

\section{References}

Brdys, M., \& Ulanicki, B. (1994). Operational Control of Water Systems: Structures, algorithms and applications. UK: Prentice Hall International.

Brdys, M. A., Grochowski, M., Gminski, T., Konarczak, K., \& Drewa, M. (2008). Hierarchical predictive control of integrated wastewater treatment systems. Control Engineering Practice, 16, 751-767.

Brdys, M. A., \& Tatjewski, P. (2005). Iterative Algorithms for Multilayer Optimizing Control. London: Imperial College Press. 
Cembrano, G., Quevedo, J., Puig, V., Perez, R., Figueras, J., Ramon, G., Rodryguez, P., Barnet, G., Casas, M., Verdejo, J. M., Gil, A., Marti, J., \& Konig, H. (2005). Predictive optimal control of water supply and distribution. Control Engineering Practice, .

Cembrano, G., Quevedo, J., Salamero, M., Puig, V., Figueras, J., \& Martí, J. (2004). Optimal control of urban drainage systems: a case study. Control Engineering Practice, 12, 1-9.

Charnes, A., \& Cooper, W. (1962). Chance constraints and normal deviates. Journal of the American Statistical Association, 57, 134-148.

Cheng, X., \& Krogh, B. H. (2001). Stability-constrained model predictive control. IEEE Trans. Automat. Contr., 46, 1816-1820.

Chisci, L., Falugi, P., \& Zappa, G. (2001). Predictive control for constrained systems with polytopic uncertainty. Proc. of the American Control Conference, 4, 3073-3078.

De Nicolao, L. M., \& Scattolini, R. (1998). Stabilizing receding-horizon control of nonlinear time-varying systems. IEEE Trans. Automat. Contr., 43, 10301036.

Evans, R., Li, L., Mareels, I., Okello, N., Pham, M., Qiu, W., \& Saleem, S. K. (2011). Real-time optimal control of river basin networks. Preprints of the 18th IFAC World Congress, (pp. 11459-11464).

G.Calafiore, \& F.Dabbene (2006). Probabilistic and randomized methods for design under uncertainty. Springer. 
Genceli, H., \& Nikolaou, M. (1993). Robust stability analysis of constrained norm model predictive control. AIChE J., 39, 1954-1965.

Goulart, P. J., Kerrigan, E. C., \& Maciejowski, J. M. (2006). Optimization over state feedback policies for robust control with constraints. Automatica, 42, $523-533$.

Kwakernaak, H., \& Sivan, R. (1972). Linear Optimal Control Systems. New York:Wiley.

Kwon, W. H., \& Byun, D. G. (1989). Receding horizon tracking control as a predictive control and its stability properties. Int. J. Control, 50, 1807-1824.

Kwon, W. H., \& Pearson, A. E. (1977). A modified quadratic cost problem and feedback stabilization of a linear system. IEEE Trans. Automat. Contr., AC-22, $838-842$.

Lee, J., \& Yu, Z. (1997). Worse-case formulations of model predictive control for systems with bounded parameters. Automatica, 33, 763-781.

Lee, T., Wang, Q., \& Tan, K. (1996). Robust Smith-predictor controller for uncertain delay systems. AIChE Journal, 42.

Litrico, X., \& Fromion, V. (2004). Simplified modeling of irrigation canals for controller design. Journal of Irrigation and Drainage Engineering, 130, 373 383.

Maciejowski, J. M. (2002). Predictive Control with Constraints. Great Britain: Prentice Hall. 
Marinaki, M., \& Papageorgiou, M. (2005). Optimal Real-time Control of Sewer Networks. London: Springer.

Mayne, D., \& Michalska, H. (1990). Receding horizon control of nonlinear systems. IEEE Trans. Automat. Contr., 35, 814-824.

Mayne, D., Rawlings, J., Rao, C., \& Scokaert, P. (2000). Constrained model predictive control: Stability and optimality. Automatica, 36, 789-814.

Mays, L. (2004). Urban Stormwater Management Tools. USA: McGrawHill Professional Publishing.

Negenborn, R. R. (2008). Multi-Agent Model Predictive Control with Applications to Power Networks. Delft, The Netherlands: Delft University Press.

Ocampo-Martinez, C., Puig, V., Cembrano, G., \& Quevedo, J. (2013). Application of predictive control strategies to the management of complex networks in the urban water cycle. IEEE Control Systems Magazine, 33, 15-45.

Overloop, P. J. V. (2006). Model Predictive Control on Open Water Systems. Delft, The Netherlands: Delft University Press.

Quevedo, J., Puig, V., Cembrano, G., \& Blanch, J. (2010). Validation and reconstruction of flow meter data in the Barcelona water distribution network. Control Engineering Practice, 11, 640-651.

Rakovic, S., B.Kouvaritakis, M.Cannon, C.Panos, \& R.Findeisen (2012). Parameterized tube model predictive control. IEEE Transactions on Automatic Control, 57, 2746-2761. 
Rawlings, J. B., \& Muske, K. R. (1993). Stability of constrained receding horizon control. IEEE Trans. Automat. Contr., 38, 1512-1516.

R.Scattolini (2009). Architectures for distributed and hierarchical model predictive control: A review. Journal of Process Control, 19, 723-731.

Shapiro, A., Dentcheva, D., \& Ruszczynski, A. (2009). Lectures on stochastic programming: modeling and theory. Society for Industrial and Applied Mathmatics and Mathematical Programming Society.

Tatjewski, P. (2008). Advanced control and on-line process optimization in multilayer structures. Annual Reviews of Control, 32, 71-85.

Thomas, Y. A. (1975). Linear quadratic optimal estimation and control with receding horizon. Electron. Lett., 11, 19-21.

Toro, R., Ocampo-Martinez, C., Logist, F., Impe, J. V., \& Puig, V. (2011). Tuning of predictive controllers for drinking water networked systems. Proc. 18th IFAC World Congress, . 